\title{
Colossal enhancement of spin-orbit coupling in weakly hydrogenated graphene
}

\author{
Jayakumar Balakrishnan ${ }^{1,2 \dagger}$, Gavin Kok Wai Koon ${ }^{1,2,3 \dagger}$, Manu Jaiswal ${ }^{1,2 \dagger}$, A. H. Castro Neto ${ }^{1,2,4}$ \\ and Barbaros Özyilmaz, ${ }^{1,2,3,4 \star}$
}

\begin{abstract}
Graphene's extremely small intrinsic spin-orbit (SO) interaction ${ }^{1}$ makes the realization of many interesting phenomena such as topological/quantum spin Hall states ${ }^{2,3}$ and the spin Hall effect ${ }^{4}$ (SHE) practically impossible. Recently, it was predicted ${ }^{1,5-7}$ that the introduction of adatoms in graphene would enhance the SO interaction by the conversion of $s p^{2}$ to $s p^{3}$ bonds. However, introducing adatoms and yet keeping graphene metallic, that is, without creating electronic (Anderson) localization ${ }^{8}$, is experimentally challenging. Here, we show that the controlled addition of small amounts of covalently bonded hydrogen atoms is sufficient to induce a colossal enhancement of the SO interaction by three orders of magnitude. This results in a SHE at zero external magnetic fields at room temperature, with non-local spin signals up to $100 \Omega$; orders of magnitude larger than in metals 9 . The non-local SHE is, further, directly confirmed by Larmor spin-precession measurements. From this and the length dependence of the non-local signal we extract a spin relaxation length of $\sim 1 \mu \mathrm{m}$, a spin relaxation time of $\sim 90 \mathrm{ps}$ and a SO strength of $\mathbf{2 . 5} \mathrm{meV}$.
\end{abstract}

Graphene $^{10}$ is an ideal two-dimensional (2D) system with large Young's modulus $^{11}$ and low bending rigidity ${ }^{12}$. Its extraordinary in-plane mechanical strength allows for large out-of-plane deformations, even at the atomic scale. This enables a broad class of chemical reactions/functionalizations, that are not practical with other 2D materials ${ }^{13-15}$. The out-of-plane distortion of the planar carbon bonds is unique to graphene and may allow for a strong enhancement in its otherwise weak intrinsic SO coupling strength ${ }^{1}$. This enhancement is unlike the SO enhancement in metals ${ }^{16}$ and semiconductors ${ }^{17}$, and is even distinct from the curvatureinduced SO coupling in carbon nanotubes ${ }^{18,19}$. As the $s p^{3}$-bond angle depends strongly on the graphene-substrate interaction, the hydrogenation of graphene allows for a controllable SO strength ranging from a few tens of microelectronvolts up to $7 \mathrm{meV}$ (ref. 1). This allows the manipulation of electron/hole spins in graphene through SHE (refs 17,20-24), thus eliminating the need for any magnetic elements or externally applied (local) magnetic fields in the device architecture.

We introduce small amounts of covalently bonded hydrogen atoms to the graphene lattice by the dissociation of a hydrogen silsesquioxane (HSQ) resist ${ }^{25}$. The extent of hydrogenation for our samples is determined by Raman spectroscopy measurements ${ }^{26,27}$ (see Supplementary Information) and gives $\sim 0.01-0.05 \%$ hydrogenation for a HSQ dose in the range $0.4-5 \mathrm{mC} \mathrm{cm}^{-2}$
(Fig. 1c). Our studies focus on samples that are only weakly hydrogenated, because hydrogen atoms are predicted to cluster at higher densities ${ }^{28}$. In such samples, our spin-transport measurements both at room temperature and low temperatures show a large non-local signal in the absence of any externally applied magnetic fields. By studying the length, width, adatom density and in-plane magnetic field dependence of the spin signal, we estimate the SO coupling strength $\left(\Delta_{\mathrm{SO}}\right)$, the spin relaxation length $\left(\lambda_{\mathrm{s}}\right)$ and the spin relaxation time $\left(\tau_{\mathrm{s}}\right)$.

Charge and spin transport measurements are characterized in graphene Hall bar devices (Fig. 1 and see Supplementary Information). The scanning electron micrograph of one such device with multiple Hall bar junctions is shown in Fig. 1a. The room-temperature local resistivity $(\rho)$ and the non-local resistance $\left(R_{\mathrm{NL}}\right)$ measurements for the exfoliated pristine graphene device S1 with length $L=2 \mu \mathrm{m}$ and width $W=1 \mu \mathrm{m}$ are shown in Fig. 2a. The presence of a finite $R_{\mathrm{NL}}$ at zero fields is not intriguing, because it is comparable to the estimated ohmic contribution ( $R_{\text {Ohmic }}$; refs 29,30),

$$
R_{\mathrm{Ohmic}}=\rho \mathrm{e}^{-\pi L / W}
$$

However, already after very weak hydrogenation $\sim 0.02 \%$, we observe a significant $(\sim 400 \%)$ increase in $R_{\mathrm{NL}}$ (Fig. $2 \mathrm{a}$ ), well above what can be accounted for by $R_{\text {Ohmic }}$. With increasing hydrogenation the measured $R_{\mathrm{NL}}$ shows a steep increase, reaching $170 \Omega$ at $0.05 \%$ hydrogenation (Fig. 2 b). A strong increase of the $R_{\mathrm{NL}}$ is observed even at charge densities $>1 \times 10^{12} \mathrm{~cm}^{-2}$. These results are reproduced consistently in 18 junctions in 5 samples. As the ohmic contribution to $R_{\mathrm{NL}}$ remains negligible over the entire hydrogenation rate (Fig. 2b), the only plausible explanation for the observed physical phenomenon (in the absence of an applied field and at room temperature) is the SHE.

The most direct way to confirm the SHE is to study the in-plane magnetic field sweeps, where only the presence of spin-polarized current can lead to an oscillating signal ${ }^{29}$. For this geometry the non-local signal has been predicted to oscillate in a magnetic field range given by the Larmor frequency $\omega_{B}=\Gamma B \leq\left(D_{s} / W^{2}\right)$ (ref. 29), where $\Gamma$ is the gyromagnetic ratio, $B$ is the applied magnetic field, $D_{\mathrm{s}}$ is the spin diffusion coefficient and $W$ is the width of the sample. For this, devices with higher mobility (higher $D$ ) and smaller $W$ are selected so that the condition $W<\lambda_{\mathrm{s}}$ is satisfied and the variation in the spin polarization across the strip is negligible ${ }^{29}$. Figure 3 shows the in-plane field dependence of $R_{\mathrm{NL}}$ for the device with $0.01 \%$

\footnotetext{
${ }^{1}$ Department of Physics, 2 Science Drive 3, National University of Singapore, Singapore 117542, Singapore, ${ }^{2}$ Graphene Research Centre, 6 Science Drive 2 , National University of Singapore, Singapore 117546, Singapore, ${ }^{3}$ Nanocore, 4 Engineering Drive 3, National University of Singapore, Singapore 117576, Singapore, ${ }^{4}$ NUS Graduate School for Integrative Sciences and Engineering (NGS), Centre for Life Sciences (CeLS), 28 Medical Drive, Singapore 117456, Singapore. These authors contributed equally to this work. Present address: Department of Physics, Indian Institute of Technology Madras, Chennai 600036, India. ^e-mail: barbaros@nus.edu.sg.
} 

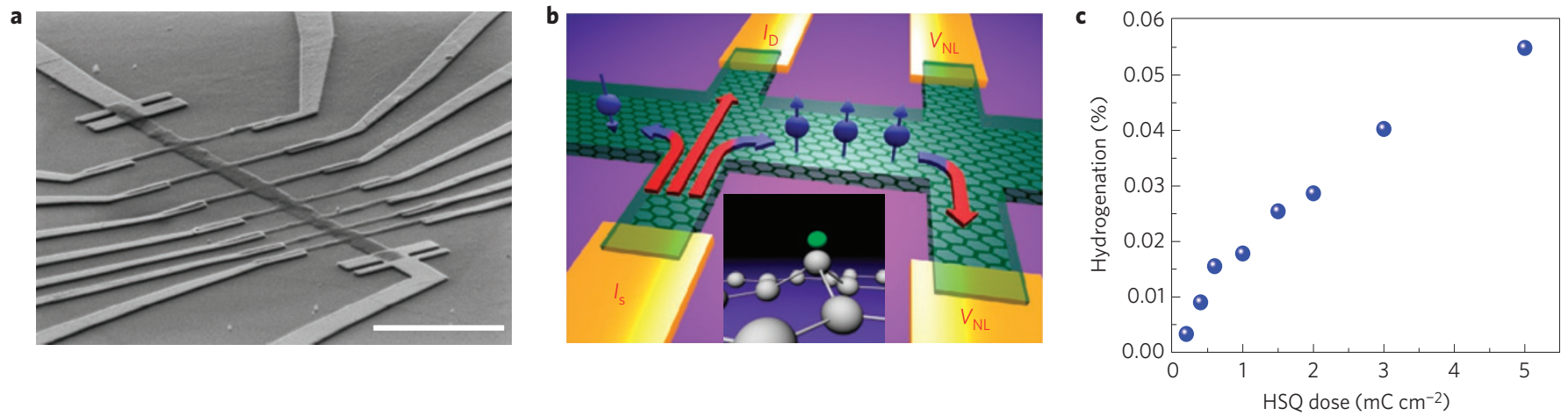

Figure 1 | Device characterization. a, Scanning electron micrograph of a hydrogenated graphene sample showing multiple Hall bar junctions. Scale bar, $5 \mu \mathrm{m}$. b. Measurement schematics for the non-local spin Hall measurement. Inset: schematics showing the deformation of the graphene hexagonal lattice

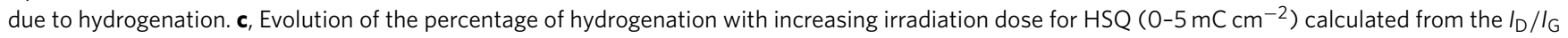
ratio of Raman peaks.

a

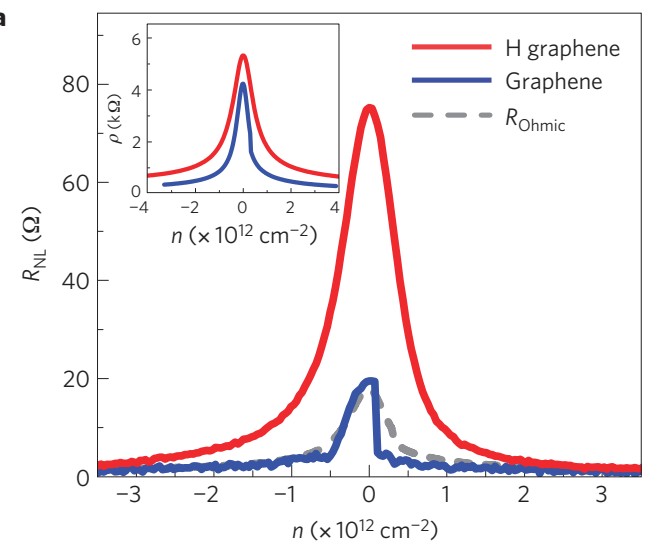

b

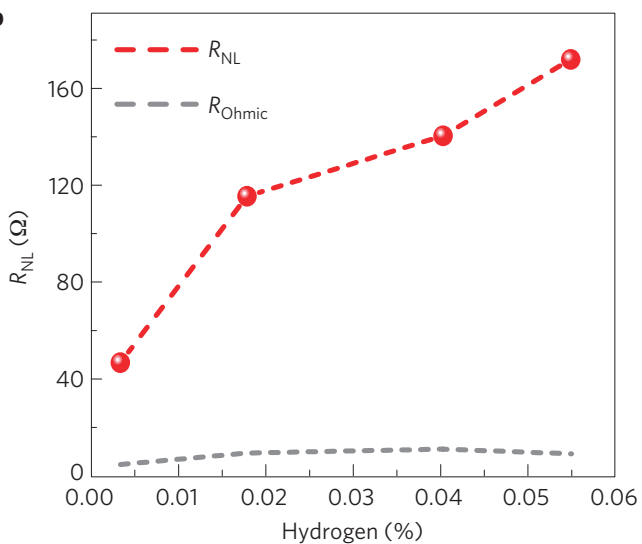

Figure 2 | Room-temperature measurements of non-local signal. $\mathbf{a}, R_{\mathrm{NL}}$ versus $n$ for pristine graphene and hydrogenated graphene at room temperature. The dark grey dashed lines show the ohmic contribution to the measured signal. Inset: $\rho$ versus $n$ for pristine and hydrogenated graphene. $\mathbf{b}$, Dependence of the $R_{N L}$ on the percentage of hydrogenation. The dark grey dashed lines show the calculated $R_{\text {Ohmic }}$ contribution for this sample.

hydrogenation at $T=4 \mathrm{~K}\left(\mu \sim 20,000 \mathrm{~cm}^{2} \mathrm{~V}^{-1} \mathrm{~s}^{-1}, L / W=5\right)$. A fit to this oscillating non-local signal using ${ }^{29}$

$$
R_{\mathrm{NL}}=\frac{1}{2} \gamma^{2} \rho W \operatorname{Re}\left[\left(\sqrt{1+i \omega_{B} \tau_{\mathrm{s}}} / \lambda_{\mathrm{s}}\right) \mathrm{e}^{-\left(\sqrt{1+i \omega_{B} \tau_{\mathrm{s}}} / \lambda_{\mathrm{s}}\right)|L|}\right]
$$

where $\gamma$ is the spin Hall coefficient, gives $\lambda_{\mathrm{s}}(B) \sim 1.6 \mu \mathrm{m}$ and $\gamma \sim 0.18$. It should be noted that such an oscillatory behaviour is absent for pristine graphene samples. Thus, the oscillatory behaviour of $R_{\mathrm{NL}}$ is a direct signature of both the SHE arising from the hydrogenation of the graphene lattice and the enhancement of an otherwise weak SO coupling strength on hydrogenation.

Further to the magnetic field dependence, we also employed the length and the width dependence to confirm that the origin of the non-local signal in weakly hydrogenated graphene samples is due to the SHE. We first discuss the length dependence by keeping $W=$ $1 \mu \mathrm{m}$ constant. Figure $4 \mathrm{a}$,b shows the length dependence of $R_{\mathrm{NL}} / \rho$, both at the charge neutrality point (CNP) and at $n=1 \times 10^{12} \mathrm{~cm}^{-2}$, for the same sample (S3) hydrogenated first to $0.02 \%$ and then at $0.05 \%$. The sample has mobilities of $1600 \mathrm{~cm}^{2} \mathrm{~V}^{-1} \mathrm{~s}^{-1}$ and $900 \mathrm{~cm}^{2} \mathrm{~V}^{-1} \mathrm{~s}^{-1}$ for $0.02 \%$ and $0.05 \%$ hydrogenation respectively. At zero applied field the equation (1) for the non-local signal, for a device with length $L$ and width $W$, becomes ${ }^{29,30}$

$$
R_{\mathrm{NL}}=\frac{1}{2} \gamma^{2} \rho \frac{W}{\lambda_{\mathrm{s}}} \mathrm{e}^{-L / \lambda_{\mathrm{s}}}
$$

By fitting the $R_{\mathrm{NL}} / \rho$ versus $L$ curve using equation (2), we determine $\lambda_{\mathrm{s}} \sim(0.95 \pm 0.02) \mu \mathrm{m}$ and $\gamma \sim 0.58$ at CNP and $\lambda_{\mathrm{s}} \sim$ $(1.12 \pm 0.06) \mu \mathrm{m}$ and $\gamma=0.45$ at $n=1 \times 10^{12} \mathrm{~cm}^{-2}$. These results are consistent and in good agreement with the results from conventional lateral spin-valve ${ }^{31-34}$ devices for hydrogenated graphene with ferromagnetic contacts ${ }^{35}$ (see Supplementary Information for further data).

Next we study the width dependence of the non-local signal at a fixed length $L=2 \mu \mathrm{m}$ (Fig. 4c, sample S2). After $0.01 \%$ hydrogenation S2 still shows a mobility of $14,000 \mathrm{~cm}^{-2} \mathrm{~V}^{-1} \mathrm{~s}^{-1}$ at room temperature. In such higher mobility samples, the width dependence of the SHE signal shows a power-law dependence. The $R_{\text {Ohmic }}$, on the other hand, depends on the width as $\exp (-\pi L / W)$ and is orders of magnitude smaller. The distinction between $R_{\mathrm{NL}}$ and $R_{\text {Ohmic }}$ is most apparent at the smallest width $(400 \mathrm{~nm})$. This is in good agreement with the theoretical prediction of ref. 29, for narrow channels. The observed width dependence can also be well explained by the theoretical model for clean wires ${ }^{36,37}$, that is, for high-mobility devices in the limit $W<\lambda_{\text {so }}$, where $\lambda_{\text {so }}$ is the spin-precession length. For most of our width range $(0.4-1.8 \mu \mathrm{m})$ this condition is easily fulfilled, because for S2 $\lambda_{\text {so }} \sim 8 \mu \mathrm{m}$ (see Supplementary Information).

We next evaluate other key spin parameters such as the $\tau_{\mathrm{s}}$ and the $\Delta_{\text {SO }}$. In hydrogenated graphene, the dominant spin relaxation is predicted to be the spin dephasing due to ElliottYafet scattering ${ }^{1}$. In the Elliott-Yafet mechanism, $\tau_{\mathrm{s}}=\left(\varepsilon_{\mathrm{F}} / \Delta_{\mathrm{SO}}\right)^{2} \tau_{p}$, where $\varepsilon_{\mathrm{F}}$ is the Fermi energy and $\tau_{p}$ is the momentum relaxation 


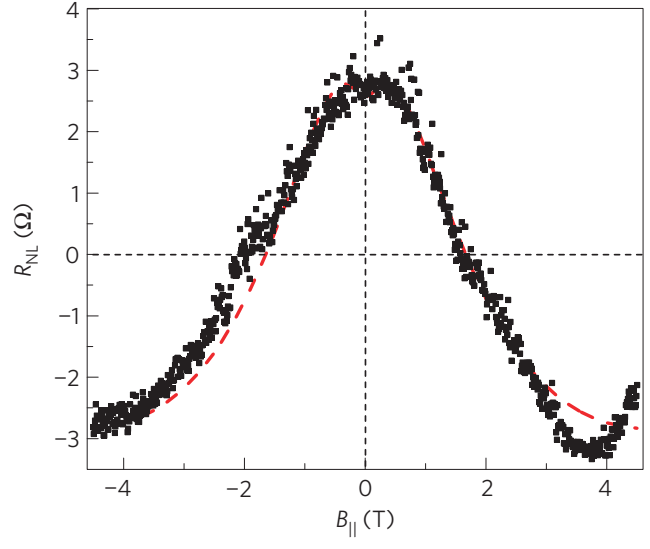

Figure 3 | Magnetic field dependence of $\boldsymbol{R}_{\mathrm{NL}}$. Parallel-field precession data for the sample S2 with $L / W=5$ and mobility $\sim 20,000 \mathrm{~cm}^{2} V^{-1} \mathrm{~s}^{-1}$. A smooth background has been subtracted from the raw data (see Supplementary Information). The red dotted line is the fit for the experimental curves. The fitting gives $\lambda_{\mathrm{s}} \sim 1.6 \mu \mathrm{m}$.

time ${ }^{38,39}$. By substituting the value for $\tau_{p}, \tau_{\mathrm{s}}=\lambda_{\mathrm{s}}^{2} / D=90 \mathrm{ps}(D$ is the diffusion coefficient from the $R$ versus $n$ curve) and the $\varepsilon_{\mathrm{F}}$ at $n=1 \times 10^{12} \mathrm{~cm}^{-2}$, we estimate $\Delta_{\mathrm{SO}}^{\mathrm{H}} \cong 2.5 \pm 0.2 \mathrm{meV}$ for hydrogenated samples. Remarkably this is three orders of magnitude higher than the value predicted for pristine graphene. In such an undistorted graphene lattice, all of the bonds are $s p^{2}$ with the $\sigma$ and $\pi$ bands orthogonal and separated by energy of the order of $\Delta_{\mathrm{E}} \cong 10 \mathrm{eV}$. Hence, SO coupling can occur only as a second-order process with energy $\Delta_{\text {SO }}^{s p^{2}} \cong\left|\Delta_{\text {SO }}^{\text {at }}\right|^{2} / \Delta_{E} \cong 10 \mu \mathrm{eV}$, explaining the small SO strength in flat graphene ${ }^{40}$. On the other hand, an adatom locally breaks the reflection symmetry across the graphene plane leading to an out-of-plane distortion by an angle $\varphi$ relative to the plane (it varies from $\varphi=0^{\circ}$ for $s p^{2}$, to $\varphi \approx 19.5^{\circ}$ for a full $\left.s p^{3}\right)$. For $\varphi \neq 0^{\circ}$ the distortion mixes $\sigma$ and $\pi$ orbitals that are no longer orthogonal (for full $s p^{3}$ these states are degenerate). Hence, the SO interaction becomes a first-order effect leading to a large enhancement of SO coupling for covalently bonded hydrogen impurities in graphene. Following ref. 1, we can show that the strength of the SO coupling can be written as: $r_{\mathrm{SO}}=\Delta_{\mathrm{SO}}^{\mathrm{H}} / \Delta_{\mathrm{SO}}^{\text {at }} \cong-3 \tan (\varphi)\left[1-2 \tan ^{2}(\varphi)\right]^{1 / 2}$ or equivalently, $\varphi=\operatorname{Arctan}\left\{\left[1 / 4-\left(9-8 r_{\mathrm{SO}}^{2}\right)^{1 / 2} / 12\right]^{1 / 2}\right\}$. Our experimental results give $r_{\mathrm{SO}} \cong 0.25$, which implies $\varphi \approx 5^{\circ}$. This value is of the same order of magnitude as the value predicted theoretically, $\varphi_{\mathrm{DFT}} \approx 16^{\circ}$, by $a b$ initio calculations for suspended graphene $e^{41}$. We assign the difference between the measured value and the one obtained by density function theory (DFT) to the interaction between graphene and its substrate ${ }^{40}$, which is not taken into account in $a b$ initio methods.

In the presence of this local SO coupling two new terms can be added to the Hamiltonian $H_{0}=-i \hbar v_{\mathrm{F}} \sigma \cdot \nabla$, where $\sigma$ are Pauli matrices that act on the subspace of the sublattices $\mathrm{A}$ and $\mathrm{B}, v_{\mathrm{F}}$ is the Fermi-Dirac velocity $\left(\sim 10^{6} \mathrm{~m} \mathrm{~s}^{-1}\right)$ and $\nabla$ is the gradient operator: a Dresselhaus term, $H_{\mathrm{D}}=\lambda_{\mathrm{D}} \sigma_{z} s_{z} \tau_{z} \delta(\mathbf{r}) a^{2}$, where $\tau_{z}= \pm 1$ represents either the K or $\mathrm{K}^{\prime}$ points ( $a$ is the lattice spacing); and a Rashba term, $H_{\mathrm{R}}=2 \lambda_{\mathrm{R}}\left(\sigma_{x} s_{y} \tau_{z}-\sigma_{x} s_{y}\right) \delta(r) a^{2}$. Note that $H_{\mathrm{D}}$ is invariant under time-reversal symmetry, $T(T: \sigma \rightarrow \sigma, T: s \rightarrow-s, T: \tau \rightarrow-\tau)$ and parity, $P(P: \sigma \rightarrow-\sigma, P: s \rightarrow s, P: \tau \rightarrow-\tau)$. For a uniform system, it opens a gap in the spectrum of size $\lambda_{\mathrm{D}} . H_{\mathrm{R}}$ is allowed in this case because reflection symmetry is broken. In a uniform system, this term does not open a gap but it splits the up and down spin bands into four hyperbolic bands of energy $E_{\mathrm{R}}(k)= \pm \lambda_{\mathrm{R}} \pm\left[\lambda_{\mathrm{R}}^{2}+v_{\mathrm{F}}^{2}\right]^{1 / 2}$ that are parabolic at low energies with an effective mass given by $m^{*}=\lambda_{\mathrm{R}} / v_{\mathrm{F}}^{2}$. These two terms are allowed by symmetry and, in a scattering process, lead to a spin precession and hence to SHE.

Finally, we identify the dominant microscopic mechanism for the observed spin Hall scattering ${ }^{42}$. For the side-jump mechanism the spin Hall resistivity $\rho_{\mathrm{SHE}}$ is independent of $n_{\mathrm{imp}}$, whereas for the skew-scattering mechanism $\rho_{\mathrm{SHE}} \propto n_{\text {imp }}$ (ref. 42). The spin Hall resistivity $\rho_{\mathrm{SHE}}$ is estimated from $\gamma=\left(\sigma_{\mathrm{SHE}} / \sigma_{\mathrm{G}}\right)=\left(\rho_{G} / \rho_{\mathrm{SHE}}\right)$ and gives a value of $\rho_{\text {SHE }}=12.9 \mathrm{k} \Omega$ and $14.2 \mathrm{k} \Omega$ for $0.02 \%$ and $0.05 \%$ hydrogenation, respectively. The value of $\rho_{\mathrm{SHE}}$, thus, depends only weakly on $n_{\text {imp }}\left(n_{\text {imp }} \sim 0.9 \times 10^{12} \mathrm{~cm}^{-2}\right.$ for $0.02 \%$ and $1.6 \times 10^{12} \mathrm{~cm}^{-2}$ for $0.05 \%$ hydrogenation) and hence suggests the dominance of the side-jump mechanism in hydrogenated graphene samples ${ }^{42}$.

We have demonstrated that the SO interaction in graphene can be markedly enhanced by introducing a small concentration of covalently bonded adatoms, without a significant suppression of the conductivity. Hydrogenated graphene has been used as a model system to demonstrate that this leads to a strong SHE. The SHE is confirmed by the non-monotonic oscillatory behaviour of the non-local signal in an applied in-plane magnetic field and also by the length, width and adatom density dependence of the nonlocal signal. From the length dependence of the non-local signal, we extract a spin relaxation length of $\sim 1 \mu \mathrm{m}$, a spin relaxation time of $\sim 90 \mathrm{ps}$ and a SO interaction strength of $\sim 2.5 \mathrm{meV}$ for samples with $0.05 \%$ hydrogenation. These findings are crucial for the development of graphene-based spintronics applications, as the need for magnetic elements is eliminated from the device

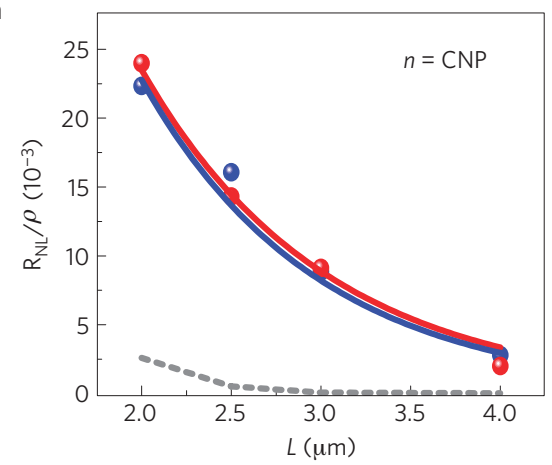

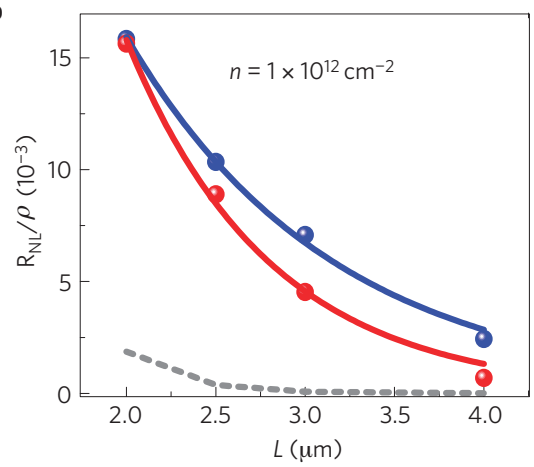

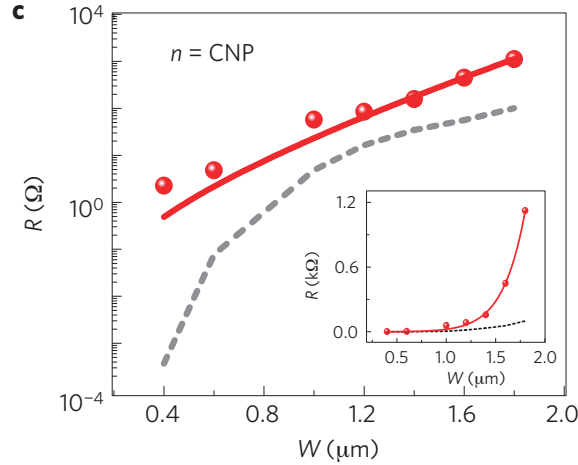

Figure 4 | Length and width dependence of $\boldsymbol{R}_{\mathbf{N L}}$ at room temperature. a,b. At the CNP (a) and at $n=1 \times 10^{12} \mathrm{~cm}{ }^{-2}(\mathbf{b})$ for samples with $0.02 \%$ (red solid circles) and $0.05 \%$ (blue solid circles) hydrogenation. Here the length is the centre-centre distance between the injector and detector electrodes. The solid lines are the fit for the data and the dark grey dashed line is the calculated ohmic contribution at these charge carrier densities. $\mathbf{c}$, $R_{\mathrm{NL}}$ (red circles) as a function of width $W(W=400 \mathrm{~nm}-1.8 \mu \mathrm{m})$ for a fixed length $L(2 \mu \mathrm{m})$. The solid red line is the fit for the data and the dark grey dashed line is the calculated ohmic contribution (inset: $R$ versus $W$ on a linear scale). 
architecture. Last but not least, the demonstration of the non-local SHE due to impurity adatoms in graphene is a major step in the realization of a robust $2 \mathrm{D}$ topological states ${ }^{6}$ and a SHE-based spin transistor at room temperature.

\section{Received 10 September 2012; accepted 8 February 2013;} published online 17 March 2013

\section{References}

1. Castro Neto, A. H. \& Guinea, F. Impurity-induced spin-orbit coupling in graphene. Phys. Rev. Lett. 103, 026804 (2009).

2. Kane, C. L. \& Mele, E. J. Z(2) topological order and the quantum spin Hall effect. Phys. Rev. Lett. 95, 146802 (2005).

3. Kane, C. L. \& Mele, E. J. Quantum spin Hall effect in graphene. Phys. Rev. Lett. 95, 226801 (2005)

4. Hirsch, J. E. Spin Hall effect. Phys. Rev. Lett. 83, 1834-1837 (1999).

5. Schmidt, M. J. \& Loss, D. Edge states and enhanced spin-orbit interaction at graphene/graphane interfaces. Phys. Rev. B 81, 165439 (2010).

6. Conan, W., Jun, H., Jason, A., Marcel, F. \& Ruqian, W. Engineering a robust quantum spin Hall state in graphene via adatom deposition. Phys. Rev. X 1, 021001 (2011).

7. Zhou, J., Liang, Q. F. \& Dong, J. M. Enhanced spin-orbit coupling in hydrogenated and fluorinated graphene. Carbon 48, 1405-1409 (2010).

8. Rappoport, T. G., Uchoa, B. \& Castro Neto, A. H. Magnetism and magnetotransport in disordered graphene. Phys. Rev. B 80, 245408 (2009).

9. Seki, T. et al. Giant spin Hall effect in perpendicularly spin-polarized FePt/Au devices. Nature Mater. 7, 125-129 (2008).

10. Novoselov, K. S. et al. Electric field effect in atomically thin carbon films. Science 306, 666-669 (2004).

11. Lee, C., Wei, X. D., Kysar, J. W. \& Hone, J. Measurement of the elastic properties and intrinsic strength of monolayer graphene. Science 321, 385-388 (2008).

12. Kim, E. A. \& Castro Neto, A. H. Graphene as an electronic membrane. Europhys. Lett. 84, 57007 (2008).

13. Loh, K. P., Bao, Q. L., Ang, P. K. \& Yang, J. X. The chemistry of graphene. J. Mater. Chem. 20, 2277-2289 (2010).

14. Elias, D. C. et al. Control of graphene's properties by reversible hydrogenation: Evidence for graphane. Science 323, 610-613 (2009).

15. Nair, R. R. et al. Fluorographene: A two-dimensional counterpart of teflon. Small 6, 2877-2884 (2010).

16. Fert, A. \& Levy, P. M. Spin Hall effect induced by resonant scattering on impurities in metals. Phys. Rev. Lett. 106, 157208 (2011).

17. Wunderlich, J., Kaestner, B., Sinova, J. \& Jungwirth, T. Experimental observation of the spin-Hall effect in a two-dimensional spin-orbit coupled semiconductor system. Phys. Rev. Lett. 94, 047204 (2005).

18. Kuemmeth, F., Ilani, S., Ralph, D. C. \& McEuen, P. L. Coupling of spin and orbital motion of electrons in carbon nanotubes. Nature 452, 448-452 (2008).

19. Jespersen, T. S. et al. Gate-dependent spin-orbit coupling in multielectron carbon nanotubes. Nature Phys. 7, 348-353 (2011).

20. Kato, Y. K., Myers, R. C., Gossard, A. C. \& Awschalom, D. D. Observation of spin Hall effect in semiconductors. Science 306, 1910-1913 (2004).

21. Dyakonov, M. I. \& Perel, V. I. Current-induced spin orientation of electrons in semiconductors. Phys. Lett. A 35, 459-460 (1971).

22. Valenzuela, S. O. \& Tinkham, M. Direct electronic measurement of the spin Hall effect. Nature 442, 176-179 (2006).

23. Abanin, D. A. et al. Giant nonlocality near the Dirac point in graphene. Science 332, 328-330 (2011).

24. Brüne, C. et al. Spin polarization of the quantum spin Hall edge states. Nature Phys. 8, 485-490 (2012).

25. Ryu, S. et al. Reversible basal plane hydrogenation of graphene. Nano Lett. 8, 4597-4602 (2008).
26. Jaiswal, M. et al. Controlled hydrogenation of graphene sheets and nanoribbons. ACS Nano 5, 888-896 (2011).

27. Cancado, L. G. et al. Quantifying defects in graphene via Raman spectroscopy at different excitation energies. Nano Lett. 11, 3190-3196 (2011).

28. Hornekaer, L. et al. Clustering of chemisorbed H(D) atoms on the graphite (0001) surface due to preferential sticking. Phys. Rev. Lett. 97, $186102(2006)$

29. Abanin, D. A., Shytov, A. V., Levitov, L. S. \& Halperin, B. I. Nonlocal charge transport mediated by spin diffusion in the spin Hall effect regime. Phys. Rev. B 79, 035304 (2009)

30. Mihajlovic, G., Pearson, J. E., Garcia, M. A., Bader, S. D. \& Hoffmann, A. Negative nonlocal resistance in mesoscopic gold Hall bars: Absence of the giant spin Hall effect. Phys. Rev. Lett. 103, 166601 (2009).

31. Tombros, N. et al. Electronic spin transport and spin precession in single graphene layers at room temperature. Nature 448, 571-574 (2007).

32. Yang, T-Y. et al. Observation of long spin-relaxation times in bilayer graphene at room temperature. Phys. Rev. Lett. 107, 047206 (2011).

33. Avsar, A. et al. Toward wafer scale fabrication of graphene based spin valve devices. Nano Lett. 11, 2363-2368 (2011).

34. Patra, A. K. et al. Dynamic spin injection into chemical vapor deposited graphene. Appl. Phys. Lett. 101, 162407 (2012).

35. McCreary, K. M., Swartz, A. G., Han, W., Fabian, J. \& Kawakami, R. K. Magnetic moment formation in graphene detected by scattering of pure spin currents. Phys. Rev. Lett. 109, 186604 (2012).

36. Kettemann, S. Dimensional control of antilocalization and spin relaxation in quantum wires. Phys. Rev. Lett. 98, 176808 (2007).

37. Paul, W. \& Stefan, K. in Handbook of Nanophysics: Nanotubes and Nanowires Ch 28 (CRC Press, 2010).

38. Huertas-Hernando, D., Guinea, F. \& Brataas, A. Spin-orbit-mediated spin relaxation in graphene. Phys. Rev. Lett. 103, 146801 (2009).

39. Ochoa, H., Castro Neto, A. H. \& Guinea, F. Elliot-Yafet mechanism in graphene. Phys. Rev. Lett. 108, 206808 (2012).

40. Konschuh, S., Gmitra, M. \& Fabian, J. Tight-binding theory of the spin-orbit coupling in graphene. Phys. Rev. B 82, 245412 (2010).

41. Duplock, E. J., Scheffler, M. \& Lindan, P. J. D. Hallmark of perfect graphene. Phys. Rev. Lett. 92, 225502 (2004).

42. Maekawa, S. (ed.) in Concepts in Spin Electronics Ch. 8, 363-367 (Oxford Univ. Press, 2006)

\section{Acknowledgements}

We thank A. Avsar, A. Pachoud, J. You and M. A. Cazalilla for their help and useful discussions. This work was supported by the Singapore National Research Foundation Fellowship award (RF2008-07-R-144-000-245-281), the NRF-CRP award 'Novel 2D materials with tailored properties: beyond graphene' (R-144-000-295-281) and the Singapore Millennium Foundation-NUS Research Horizons award (R-144-001-271-592; R-144-001-271-646).

\section{Author contributions}

B.Ö. devised and supervised the project. J.B. and B.Ö. designed the experiments. J.B. and G.K.W.K. performed the experiments. A.H.C.N. provided the theoretical work. All authors carried out the data analysis and discussed the results. J.B., A.H.C.N. and B.Ö. co-wrote the paper.

\section{Additional information}

Supplementary information is available in the online version of the paper. Reprints and permissions information is available online at www.nature.com/reprints. Correspondence and requests for materials should be addressed to B.Ö.

\section{Competing financial interests}

The authors declare no competing financial interests. 